\title{
Eurasian jays do not copy the choices of conspecifics, but they do show evidence of stimulus enhancement
}

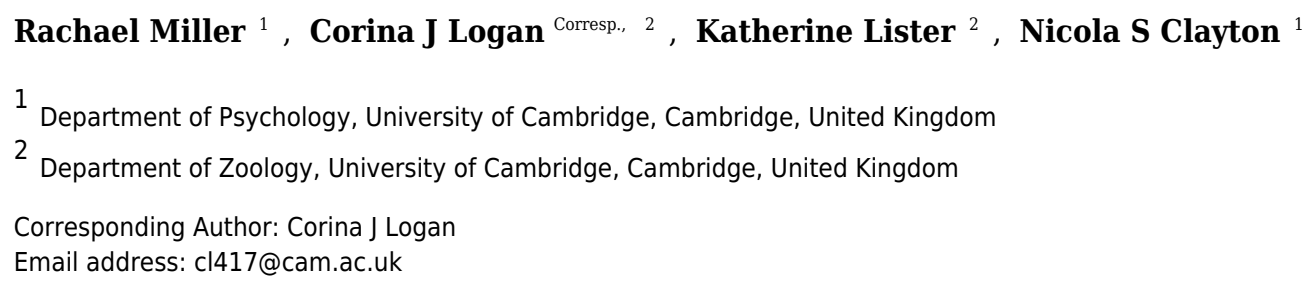

Corvids (birds in the crow family) are hypothesised to have a general cognitive tool-kit because they show a wide range of transferrable skills across social, physical and temporal tasks, despite differences in socioecology. However, it is unknown whether relatively asocial corvids differ from social corvids in their use of social information in the context of copying the choices of others, because only one such test has been conducted in a relatively asocial corvid. We investigated whether relatively asocial Eurasian jays (Garrulus glandarius) use social information (i.e., information made available by others). Previous studies have indicated that jays attend to social context in their caching and mate provisioning behaviour; however, it is unknown whether jays copy the choices of others. We tested the jays in two different tasks varying in difficulty, where social corvid species have demonstrated social information use in both tasks. Firstly, an object-dropping task was conducted requiring objects to be dropped down a tube to release a food reward from a collapsible platform, which corvids can learn through explicit training. Only one rook and one New Caledonian crow have learned the task using social information from a demonstrator. Secondly, we tested the birds on a simple colour discrimination task, which should be easy to solve, because it has been shown that corvids can make colour discriminations. Using the same colour discrimination task in a previous study, all common ravens and carrion crows copied the demonstrator. After observing a conspecific demonstrator, none of the jays solved the object-dropping task, though all jays were subsequently able to learn to solve the task in a non-social situation through explicit training, and jays chose the demonstrated colour at chance levels. Our results suggest that social and relatively asocial corvids differ in social information use, indicating that relatively asocial species may have secondarily lost this ability due to lack of selection pressure from an asocial environment. 
1 Eurasian jays do not copy the choices of conspecifics, but they do show evidence of stimulus

2 enhancement

3

4 Rachael Miller ${ }^{1}$, Corina J. Logan ${ }^{2 *}$, Katherine Lister $^{2}$ \& Nicola S. Clayton ${ }^{1}$

5

6 Affiliations:

$7 \quad{ }^{1}$ Department of Psychology, University of Cambridge, Cambridge, UK

$8 \quad 2$ Department of Zoology, University of Cambridge, Cambridge, UK

9

$10 *$ Correspondence:

11 Corina Logan (c1417@cam.ac.uk) 


\section{ABSTRACT}

14 Corvids (birds in the crow family) are hypothesised to have a general cognitive tool-kit because they show a wide range of transferrable skills across social, physical and temporal tasks, despite differences in socioecology. However, it is unknown whether relatively asocial corvids differ from social corvids in their use of social information in the context of copying the choices of others, because only one such test has been conducted in a relatively asocial corvid. We investigated whether relatively asocial Eurasian jays (Garrulus glandarius) use social information (i.e., information made available by others). Previous studies have indicated that jays attend to social context in their caching and mate provisioning behaviour; however, it is unknown whether jays copy the choices of others. We tested the jays in two different tasks varying in difficulty, where social corvid species have demonstrated social information use in both tasks. Firstly, an object-dropping task was conducted requiring objects to be dropped down a tube to release a food reward from a collapsible platform, which corvids can learn through explicit training. Only one rook and one New Caledonian crow have learned the task using social information from a demonstrator. Secondly, we tested the birds on a simple colour discrimination task, which should be easy to solve, because it has been shown that corvids can make colour discriminations. Using the same colour discrimination task in a previous study, all common ravens and carrion crows copied the demonstrator. After observing a conspecific demonstrator, none of the jays solved the object-dropping task, though all jays were subsequently able to learn to solve the task in a non-social situation through explicit training, and jays chose the demonstrated colour at chance levels. Our results suggest that social and relatively asocial corvids differ in social information use, indicating that relatively asocial species may have secondarily lost this ability due to lack of selection pressure from an asocial environment. 


\section{Introduction}

A wide range of corvid species (e.g., crows, ravens, jays) are known for their complex cognitive abilities, which are hypothesised to have been present in their common ancestor, thus forming a 'general cognitive tool-kit' across this taxa (Emery \& Clayton, 2004). For example, even though rooks (Corvus frugilegus) do not make or use tools in the wild, they are able to spontaneously innovate these behaviours in the lab (Bird \& Emery, 2009b). Further, there is evidence that some corvid species show cognitive competence across a wide range of social, physical and temporal tasks. For instance, California scrub-jays (Aphelocoma californica) show proficiency in cognitive tasks relating to memory (Clayton \& Dickinson, 1998), future planning (Clayton, Emery \& Dickinson, 2006; Raby et al. 2007), and social cognition through cache protection tactics (Clayton, Dally \& Emery, 2007). As another example, rooks, in addition to their tool abilities, cooperate with each other to solve novel problems (Seed et al. 2008) and appear to understand support relationships because they look longer at impossible scenarios (e.g., a ball suspended in mid-air rather than sitting on a table; Bird \& Emery 2010). Additionally, New Caledonian crows (Corvus moneduloides) reason about hidden causal agents (Taylor, Miller \& Gray 2012), reason by exclusion (Jelbert et al. 2015), and learn socially about novel foraging problems (Logan et al. 2016).

It is unknown whether this cognitive tool-kit includes the ability to use social information specifically in the form of copying the choices of others, which is distinct from changing behaviour when solving problems in different social contexts (several examples are given below). The corvid common ancestor is hypothesised to have been social (Clayton \& Emery, 2007). If this assumption is correct, rather than the common ancestor being asocial with sociality having evolved several times in extant lineages, then there is reason to expect that relatively 
59

60

61

62

63

64

65

66

67

68

asocial corvids could have retained the capacity to use social information. For example, it could be adaptive by improving foraging and mate searching efficiency (e.g., Valone \& Templeton, 2002). Alternatively, this ability could have been secondarily lost because of the lack of selection pressure from an asocial environment, in a similar manner to the secondary loss of caching (food hiding) in jackdaws (Corvus monedula; de Kort \& Clayton, 2006). For example, in the absence of conspecifics for most of the year, there might have been an increased selection pressure to rely solely on personal information when foraging.

Most studies of corvid social information use, in the form of copying the choices of others, have occurred in social species (species that live in groups of at least pairs year-round), which makes it difficult to determine whether this ability is part of their general cognitive tool-kit. Evidence of social information use, specifically copying the choices of others, has been found in social corvid species, including pinyon jays (Gymnorhinus cyanocephalus; Templeton, Kamil \& Balda, 1999), rooks (Dally, Clayton \& Emery, 2008), jackdaws (Corvus monedula; Schwab, Bugnyar \& Kotrschal, 2008a), common ravens (Corvus corax; Fritz \& Kotrschal, 1999; Schwab et al., 2008b), carrion crows (Corvus corone corone, C. c. cornix; Miller, Schwab \& Bugnyar, 2016) and New Caledonian crows (Logan et al., 2016). Social species are predicted to be better at acquiring new skills in a social context than in a non-social context (Lefebvre \& Giraldeau 1996), because they may attend more to conspecifics than asocial species (Balda, Kamil \& Bednekoff, 1997).

However, we are aware of only two tests of social information use in the form of copying the choices of others in a relatively asocial corvid. Clark's nutcrackers (Nucifraga columbiana) did not learn a motor or a discrimination task faster in a social learning condition than in an individual learning condition, indicating that they did not use social information (Templeton, 
82 Kamil \& Balda, 1999). This was in contrast with highly social pinyon jays that did learn faster in

83 the social learning conditions (Templeton, Kamil \& Balda, 1999). Additionally, Clark's

84 nutcrackers more accurately recovered caches they made rather than caches they observed others

85 make, in contrast with social Mexican jays that were accurate in both conditions (Bednekoff \&

86 Balda 1996). These results suggest that relatively asocial corvids attend less to social information

87 than social corvids.

88 Outside of corvids, social learning in the form of copying conspecifics has been found in a number of asocial species including red-footed tortoises (Geochelone carbonaria; Wilkinson et al. 2010), black river stingrays (Potamotrygon falkneri; Thonhauser et al. 2013; Garrone Neto \& Uieda 2012), bearded dragons (Pogona vitticeps; Kis, Huber \& Wilkinson 2014), and in juvenile, but not adult, golden hamsters (Mesocricetus auratus; Lupfer, Frieman \& Coonfield 2003) and eastern water skinks (Eulamprus quoyii; Noble, Byrne \& Whiting 2014). These non-corvid species are likely to have had asocial ancestors, which suggests that social cues are not costly to attend to and can evolve outside of a social context in these taxa. However, at present, the sample size of the relatively asocial corvid species is too small to draw general conclusions about the influence of a corvid's social system on their use of social information. We addressed this gap by investigating whether the relatively asocial Eurasian jays (Garrulus glandarius) used social information provided by a conspecific. Eurasian jays do not live in social groups except during the breeding season when mated pairs defend a territory (Goodwin 1951; Snow \& Perrins, 1997; Clayton \& Emery, 2007). There is evidence that socially housed Eurasian jays attend to social context to modify their caching and mate provisioning (courtship feeding) behaviour. For example, they prefer to cache in quiet rather than noisy substrates when in the presence of conspecifics that could hear but not see the subject (Shaw \& 
105 Clayton, 2013); they attend to spatial and auditory cues when competitors are caching to later 106 pilfer those caches (Shaw \& Clayton, 2014); and subordinates inhibit caching in front of 107 dominants and prefer to cache in less exposed areas (Shaw \& Clayton, 2012). They also adjust 108 their behaviour appropriately depending on whether they are caching or pilfering (Shaw \& 109 Clayton 2014), and whether they compete with a dominant or subordinate (Shaw \& Clayton 110 2012). Furthermore, they prefer to cache out-of-sight behind an opaque barrier and at a distance 111 when observed by conspecifics (Legg \& Clayton, 2014; Legg, Ostojić \& Clayton, 2016). During 112 the breeding season, males are attentive to which foods their mates might prefer based on how 113 much of which foods she has already eaten (Ostojić et al., 2013; Ostojić et al., 2014).

These jays were socially raised and housed, which differs from their relatively asocial 115 system in the wild. The artificially social environment likely enhances their utilisation of any 116 innate social skills because these skills will have been given the opportunity to develop from an 117 early age. Therefore, if social skills are found in these conditions, it demonstrates the potential 118 flexibility of this species to use social cues (if social cues are used). As such, the social capacities 119 shown by socially raised and housed jays might differ from wild individuals. Despite the 120 evidence that socially housed Eurasian jays can respond to social context in caching and mate provisioning paradigms, no study has yet tested whether this species uses social information to 122 copy the choices of others, which could be useful for learning about foraging opportunities even 123 in a relatively asocial species.

124 We tested whether socially housed Eurasian jays would use social information from a 125 conspecific demonstrator when learning to solve a novel problem - an object-dropping task where an object must be dropped into a tube to release a food reward from a collapsible platform.

127 Further, if the birds did not use social information to solve the task, we tested whether there was 
128 any evidence that they had attended to the demonstrator (as indicated by differences between 129 groups with differing levels of social learning opportunities), and what they might have learned 130 during this exposure. The object-dropping task has been used previously during pre-test training 131 for Aesop's Fable tasks in this species (Cheke, Bird \& Clayton, 2011) as well as in a number of 132 other bird species (rooks: Bird \& Emery, 2009a; New Caledonian crows: Jelbert et al., 2014; 133 Logan et al., 2014; California scrub-jays, Logan et al., 2016; great-tailed grackles, Quiscalus 134 mexicanus, Logan 2016). Aesop's Fable tasks require subjects to insert objects into water-filled 135 tubes to obtain out-of-reach floating rewards.

136 In the corvids that have been tested using this object-dropping task so far, we see a common pattern, irrespective of whether they are habitual tool users. Namely, they are capable of learning 138 the object-dropping task, but only once they have experienced an object falling into a tube, which usually occurs when they accidentally knock an object off the ledge into the tube. This finding suggests that the birds need to see the object fall, and once they have, they can learn to solve the rest of the task. This raises the question of whether they need direct experience of manipulating the objects and observing them fall into the tube or whether witnessing another individual's solution to the problem will suffice in learning the task. So far, only two birds have solved the object-dropping task after observing a conspecific demonstrator: one rook (Bird \& Emery, 2009b) and one New Caledonian crow (Mioduszewska, Auersperg \& von Bayern, 2015), though only the latter study aimed to explicitly test for influences of social information use on

147 learning this task. New Caledonian crows are habitual tool users in the wild (Hunt, 1996), whilst 148 rooks - like Eurasian jays - are not, though rooks have shown tool-use and manufacture 149 proficiency in the lab (Bird \& Emery, 2009b). Both rooks and crows are more social than jays, in 150 that rooks form large flocks for breeding, foraging and roosting, while New Caledonian crows 
151 tend to form extended family groups that are fairly tolerant of their neighbours (Goodwin, 1986;

152 St Clair et al., 2015).

153 We also investigated whether Eurasian jays would choose the colour that was demonstrated

154 to be rewarded in a two-choice colour discrimination test. Unlike the object-dropping task, this is

155 a fairly simple task and corvids, including Eurasian jays, have been shown to be capable of

156 making colour discriminations (ravens: Range, Bugnyar \& Kotrschal, 2008; Eurasian jays:

157 Clayton \& Krebs, 1994; Davidson et al., Under Review). Furthermore, this test has explicitly

158 been used previously to demonstrate use of social information in other corvids, namely common

159 ravens and carrion crows, where all the individuals that were tested chose the demonstrated

160 colour (Miller, Schwab \& Bugnyar, 2016). Ravens and crows are social species with high

161 fission-fusion dynamics, being highly social in non-breeding season, and territorial in the

162 breeding season (Goodwin, 1986). We conducted the task in a comparable manner to Miller,

163 Schwab \& Bugnyar (2016) to allow for direct comparison between these two corvid studies. The

164 inclusion of both tasks in the present study allowed us to compare jay performances with social

165 corvid species that have been shown to use social information on the same tasks. Furthermore,

166 the use of both tasks enabled us to control for potential influences of task affordances, such as

167 difficulty. Namely, even if the object-dropping task was too difficult to learn socially, we would

168 still be able to detect whether the jays use social information in the simpler colour-discrimination 169 task.

170 The general tool-kit hypothesis (Emery \& Clayton, 2005) may predict that relatively asocial 171 jays, like the more social New Caledonian crows, rooks, ravens and crows, would use the

172 information provided by the demonstrator, as they may have retained the capacity to use social

173 information (i.e., information made available by others). Alternatively, jays may differ from the 
174 more social corvids in their use of social information, as they may have secondarily lost this

175 ability due to lack of selection pressure from an asocial environment.

176

177 Methods

178 Subjects

179 The subjects were 16 hand-reared juvenile Eurasian jays (eight females, eight males)

180 hatched in May 2015. The birds were hand-reared as a group in 2015, and socially housed within

181 a large outdoor aviary $(9 \times 16.5 \times 6 \mathrm{~m})$ at the Sub-department of Animal Behaviour in Madingley,

182 Cambridge. Birds were sourced from wild nests at 10 days old by a registered breeder under a

183 Natural England License to NSC (20140062). The subjects consisted of five sibling groups (one

184 pair, three groups of three birds, and one group of four birds), with one individual that had no

185 siblings. Testing took place in indoor test compartments $(2 \times 2 \times 1 \mathrm{~m})$, with which the birds were

186 familiar, as they were fed their daily diet within these compartments and had constant access to

187 them outside of testing sessions. The birds could be separated individually, in pairs or sub-groups

188 within these compartments as required. One female bird ('Sjoika') did not participate in either

189 experiment, as she could not reliably be separated individually in the compartments. Subjects

190 were identifiable using unique colour leg-ring combinations. Prior to and during testing, subjects

191 had access to their daily diet, which consisted of soaked dog pellet and boiled vegetables, and

192 water. Rewards for both experiments were live mealworms, which are a highly valued food item,

193 reserved only for training and testing. Experiment 1 was conducted in October 2015 and

194 Experiment 2 in November 2015.

195

196 Animal ethics 
These experiments were conducted under approval from University of Cambridge

198

199

200

201

202

203

204

205

206

207

208

209

210

211

212

213

214

215

216

217

218

Psychology Research Ethics Committee (application number: pre.2013.109) and the European

Research Council Executive Agency Ethics Team (application: 339993-CAUSCOG-ERR).

\section{Video summary}

A video shows examples from both experiments: https://youtu.be/L3IQy8cbqUQ.

Experiment 1: Trained group, Solving Task ('Stuka'); Experiment 1: Observer Group, Test Trial 5 ('Gizmo’); Experiment 2: Observer Group, Test Trial (Gizmo).

\section{EXPERIMENT 1: Object dropping task}

\section{Materials}

The testing apparatus was a clear Perspex 'object insertion' apparatus (total height $=13 \mathrm{~cm}$ ) consisting of a tube and a box (height $=10.5 \mathrm{~cm}$, depth $=6.5 \mathrm{~cm}$, width $=11 \mathrm{~cm}$ ) containing a collapsible platform (based on the design in Bird \& Emery 2009b). Objects could be inserted into a tube (length $=8 \mathrm{~cm}$, diameter $=5 \mathrm{~cm})$, causing the collapsible platform at the bottom of the tube to release from a small magnet holding it in place. Once released from the magnet, a food reward was dispensed to the subject (Figure 1). Several clear, plastic rings and one additional removable platform $($ length $=13 \mathrm{~cm}$, width $=13 \mathrm{~cm}$ ) that attached to the exterior of the tube were used for the earlier training stages. A blue ring was added to the top of the tube to increase the salience of this area. Only one object was required to drop the collapsible platform and release the reward.

Spherical, black metal, hollow objects were used (measuring $2 \mathrm{~cm}$ in diameter and weighing 4$5 \mathrm{~g}$; Figure 1), with three thin pieces of black plastic string woven through the middle of each 
219 object and tied in a knot on each side, to allow the birds to pick up the object more easily and 220 prevent them from rolling away.

221

222 Procedure

223 Subjects were separated into three groups: a trained group that had no prior experience with 224 the apparatus and had never seen another solve it, but were trained to correctly solve it by the 225 experimenter (three males, three females); an observer group that observed a trained conspecific 226 solve the task (three males, three females); and a control group that received no training on the task and did not see any bird interact with the task (two males, one female).

\section{Habituation and spontaneous object dropping}

All subjects were habituated to the apparatus and the object separately by presenting them

231

232

233

234

235

236

237

238

239

240

241

with small food rewards on top and beside the apparatus and object. Subjects were first presented with a baited object on the table until they retrieved the reward in five consecutive trials. They were then presented with the object insertion apparatus in the stages outlined in Table 1 and Figure 1. Namely, the apparatus was presented in three scenarios to aid in learning how to correctly solve the apparatus. 1 . The removable platform was placed at the top of the tube (Figure 1a) to allow the object to be placed on the rim of the tube so the bird could easily accidentally knock the object into the tube by nudging it when attempting to obtain bait from under the object. 2. The removable platform was placed at the bottom of the tube (Figure 1b) to encourage the bird to pick up the object and lift it up to the top of the tube to insert it. 3 . The removable platform was removed (i.e., final stage apparatus; Figure 1c) so the bird had to pick up the object from the table to insert it into the top of the tube. Rewards were placed on the 
242 apparatus, as well as underneath it, with the collapsible platform in the dropped position, until

243 subjects retrieved all available rewards per trial in five consecutive trials.

244 All subjects were then given one five min test trial to determine whether they would

245 spontaneously pick up and drop the object into the tube prior to being allocated to a group.

246 During this test, the final stage apparatus (Figure 1c) was presented to each subject with the

247 object placed on the table beside the apparatus. No birds spontaneously solved the apparatus

248 within the five minutes, therefore they were randomly assigned to one of three groups: trained,

249 observer or control. Birds were allocated to groups by choosing names from a container: one

250 'male only' and one 'female only' container ensured a balanced sex ratio in each group (three

251 males, three females for the trained and observer groups; two males, one female for the control

252 group).

253

254 Trained group

255 We first trained birds in the 'trained group' to successfully solve the task by inserting

256

257

258

259

260

261

262

263

264 objects from the table into the tube and obtaining the reward. We used the training stages outlined in Table 1 and Figure 1 to gradually increase their proficiency from accidentally inserting baited objects balanced on the rim of the tube to nudging objects down the tube with the use of a removable platform attached to the outside of the tube (stages 1-2; Table 1), until they picked up objects from the table to insert into the tube without the removable platform present (stage 3; Table 1). In training stage 1, the object was baited with an insect on intermittent insertions for the first 1-2 training sessions (3-21 insertions, mean=11 insertions). A session for the trained group lasted 5-10 minutes and was not restricted to a specific number of object insertions, but rather determined by the subject's motivation and performance in that particular 
265 session. A maximum of two training sessions were run per day. An object insertion was

266 considered proficient if it was nudged or dropped directly into the tube, as opposed to being

267 knocked in accidentally by removing the baited insect, or first pushing it around on the platform

268 or dropping it onto the table from the platform.

269 Subjects moved from stage one to stage two when they had accidentally knocked the object

270 into the tube on 10 consecutive insertions (Figure 1a). The removable platform was then

271 gradually moved down the tube during stage two until the subject inserted the object from the

272 platform when it was placed at the bottom of the tube on 10 consecutive insertions (Figure $1 \mathrm{~b}$ ). If

273 subjects struggled with progression to the next stage (e.g., stopped inserting the object), they

274 returned to the previous stage, with the aim for each training session to 'end on a high' (i.e., with

275 a reward for inserting the object). A bird was considered to have solved the task when they had

276 inserted the object from the table into the final stage apparatus and obtained the reward in 10

277 consecutive insertions (Figure 1c).

278 We then selected one bird from the trained group (Homer) to demonstrate how to solve the 279 apparatus to the observer group. This bird was selected to be the demonstrator because he was 280 motivated and reliable during training (e.g., he was easy to call into the test compartments and 281 comfortable being close to humans), and solved the task during training fairly quickly. Homer 282 was 100\% accurate when he demonstrated for observers; therefore observers never saw failed 283 attempts.

\section{Observer group}

Observers saw the demonstrator successfully solve the apparatus 40 times per stage, using the following stage order: 3-1-2-3 (e.g., observers saw 40 demonstrations of stage three, then 40 
288 demonstrations of stage one, etc.; Table 1). This resulted in a total of 160 observations of

289 successful solves per observer bird. Observers were given four demonstration sessions of 10

290 solves per session per stage. The stages were the same as those used for the trained group (Table

291 1; Figure 1). As these stages facilitated the training of the trained group to solve the task, we

292 might expect that aspects of these stages are helpful for learning the task, hence including

293 demonstrations of each stage. Each demonstration session lasted approx. three min, with a

294 maximum of two sessions run per day. The demonstrations took place on a table in one

295 compartment, with the observers located in an adjacent, but separate compartment with free

296 visual access between compartments via mesh panels. There were three to four observer birds

297 per adjacent compartment and there were sufficient perches for all observers to view the

298 demonstrations at the same time. The observer group was split into two smaller sub-groups of

299 three birds per group for observations to ensure each bird had sufficient visual access of the

300 demonstrator and to reduce crowding within the test compartments. Each observer subject had

301 the opportunity to watch 16 demonstration sessions, with one or two sessions per day, ensuring

302 that each observer had ample opportunities to observe demonstrations.

303 Immediately after an observer saw 40 demonstrated solutions at a particular stage, observer

304 were visually isolated and presented with the object insertion apparatus at the final stage (i.e., no

305 removable platform and with the object on the table). They were then given one five-min test

306 trial to determine whether they had learnt to solve the task. Observer subjects received five five-

307 min test trials: one pre-demonstration test trial that all birds received to determine whether they

308 spontaneously solve the task, and observer birds received four test trials immediately after

309 observing demonstrations at each stage (stages 3-1-2-3; Table 1). Each test trial therefore took

310 place on a separate day, over a period of 15 days. During all test trials, the observer subject was 
311 presented with the final stage apparatus with the object on the table. To solve the task, the

312 subject was required to pick up the object from the table and insert it into the tube to release the

313 collapsible platform and obtain the reward. The longest time that any subject waited between

314 observing the last demonstration session of each stage and their own test trial was 10 minutes.

\section{Control group}

317 The control group did not receive any object insertion apparatus training or demonstrations, 318 and were presented with the 'final stage' object insertion apparatus the same number of times 319 that the observer group received the apparatus (i.e., five test trials). Test trials were run on the same test days as the observer group to avoid any potential differences between the groups due to age or other environmental factors.

\section{Data analysis}

All training and demonstration sessions and test trials were videotaped, as well as being live coded. We recorded the number of (accidental and proficient) insertions required for the trained group individuals to complete each training stage and solve the task (i.e., to insert an object from the table into the tube at the final apparatus stage in 10 consecutive insertions). For the observer and control groups, we recorded whether the subject solved the task (i.e., inserted an object from the table into the tube at the final apparatus stage, and interacted with the apparatus or object). object more than individuals in the control group during tests, we conducted a generalised linear model (GLM) using a Poisson distribution with a log link in R v3.2.1 (function: glm; R Core Team 2015). We combined the total number of times a bird touched the apparatus and object per trial (response variable) to examine whether it varied by trial number or group (control or 
335 observer; explanatory variables). We conducted a generalised linear mixed model (GLMM)

336 using a Poisson distribution with a log link (R package: lmerTest, function: glmer, Kuznetsova,

337 Brockhoff \& Christensen, 2015) to determine whether the observer group interacted more with

338 particular parts of the apparatus or object after having seen the demonstrator solve the task. We

339 examined whether the number of touches (response variable) varied according to the location

340 that was touched (apparatus base, apparatus tube, or object) by group (control or observer;

341 explanatory variables) with bird ID as a random effect. To examine whether observer jays

342 touched the apparatus/object sooner than control jays, we conducted the same GLMM just

343 mentioned, but with a different response variable: the latency (in seconds) to touch the apparatus

344 or object per test trial per bird.

345 To examine the level of certainty associated with each model, the respective models were

346 compared with all model combinations and their Akaike weights, which sum to one across the

347 models, considered (R package: MuMIn, function: dredge; Bates, Maechler \& Bolker, 2011). A

348 model was considered highly likely given the data if it had a high Akaike weight $(>0.89)$ relative

349 to the other models (Burnham \& Anderson, 2002).

350 Once Experiment 1 had been conducted, all of the birds in the control and observer groups

351 were trained to insert objects into the object insertion apparatus. We recorded the number of

352 (accidental and proficient) insertions required for the observer and control group to complete

353 each training stage and solve the task. We examined whether birds in the observer group solved

354 the task faster than birds in the trained or control groups using a GLM in R. The number of

355 object insertions required to complete stage three (insert the object from the table into the tube in

35610 consecutive insertions; response variable) was compared across conditions (trained, observer,

357 control; explanatory variable) using a Poisson family with a log link. 


\section{Results}

360 None of the jays solved the task spontaneously in the initial trial (i.e., prior to any training,

demonstrations or frequent exposure to the apparatus). In the trained group, all six jays learned to drop objects over a period of eight to 21 training sessions (4-11 days). In the observer group, zero of six jays learned to drop objects by observing the demonstrator. In the control group, zero of three jays learned to drop objects without training or demonstrations. Only one bird ('Gizmo’ - observer bird), on her final test trial, lifted the object high up while standing near the tube, but she did not insert it into the tube.

All observer and control subjects generally interacted with the apparatus and/or object Individuals in the observer group did not touch the apparatus or object more than individuals in the control group (mean touches=11 and 9, respectively; Table 2: Model 1). The Akaike weight for this model was very low (0.11), and it was the third ranked model, indicating a high level of uncertainty, therefore it is likely that there was not enough data for the model to draw strong conclusions, or the effects were too small to detect.

While the number of interactions decreased with increasing trial number in control individuals, there is weak evidence that observer individuals had relatively more interactions with the apparatus and object in later trials than control individuals (Table 2: Model 1). There was only weak evidence because the Akaike weight for the top-ranked model, which was the full model, was only 0.46 , indicating that there was a high degree of uncertainty in this model. There

379 was no evidence that birds in the observer group interacted more with particular parts of the apparatus or object after seeing the demonstrator solve the task compared with control birds

381 (mean touches=4 and 3, respectively; Table 2: Model 2). When comparing the latency to the first 
382 touch between control and observer groups, observer birds touched the apparatus/object

383 significantly sooner than control birds (mean=23 and 83 seconds, respectively; Table 2, Model 3;

384 Figure 3). This model was highly likely given the data because its Akaike weight was 0.99 . The

385 data in Figure 3 shows that there was no initial difference in latencies between control and

386 observer groups during their spontaneous test trial (trial 1), which was before the observer group

387 had access to social information about the apparatus. The difference between the two groups

388 occurred in trials 2-5 where, after the social demonstrations, observer latencies stayed the same,

389 while the control group's latencies increased.

$390 \quad$ Following this experiment, all nine jays in the observer and control groups underwent

391 training to drop objects over a period of 8-12 training sessions (five to seven days). Therefore,

392 the number of object insertions required to reach proficiency was compared between the trained,

393 observer, and control groups. Birds in the trained group required more insertions to solve the task

394 (i.e., to insert objects from the table into the tube of the final stage apparatus; mean insertions to

395 solve $=167$, GLM estimate $=0.39, \mathrm{SE}=0.06, \mathrm{z}=6.26, \mathrm{p}<0.001)$, than observer and control birds.

396 Birds in the observer (mean insertions to solve $=114, \mathrm{GLM}$ estimate $=0.01, \mathrm{SE}=0.07, \mathrm{z}=0.20$,

$397 \mathrm{p}=0.84$ ) and control (mean insertions to solve $=113, \mathrm{GLM}$ [intercept] estimate $=4.72, \mathrm{SE}=0.05$,

$398 \mathrm{z}=86.86, \mathrm{p}<0.001)$ groups did not differ in the number of insertions (Figure 4; ESM1 Table S1).

400 EXPERIMENT 2: Two-choice colour discrimination task

401 Materials

402 This set up consisted of two plastic cups - one black and one white (diameter $=6 \mathrm{~cm}$, 403 height $=14.5 \mathrm{~cm})$. Cups were spaced $30 \mathrm{~cm}$ apart on a wooden board $(50 \mathrm{~cm} \times 15 \mathrm{~cm})$. Each cup

404 was attached to its own metal rod so they could move up and down independently, but they were 
405 prevented from being removed entirely from the rod by a bolt. Cups could be lifted upwards to

406 reveal a hidden reward (Figure 2). Two live mealworms were placed underneath each cup.

407

408 Procedure

409 Demonstrator training

410 One bird acted as a demonstrator - 'Homer' - the same demonstrator as in Experiment 1. In 411 visual isolation from the observer group, Homer received four sessions (5-10 mins per session) 412 of 10 trials per session, where only one cup - the white cup - was baited ('demonstrated' cup) 413 and the other cup - the black cup - was locked down using the bolt so it could not be lifted. To 414 pass demonstrator training, Homer had to consistently lift only the demonstrated cup in all 10 415 consecutive trials within a session and not touch or try to lift the other cup before he could move 416 on to the demonstrations for observers. Homer touched both cups in session one and two, but 417 passed criterion in session three. He was given four training sessions in total to ensure 418 comparability with the number of demonstrator training sessions used for the carrion crows and 419 ravens in Miller, Schwab \& Bugnyar (2016). Homer chose the white cup 100\% of the time 420 during demonstrators for observers; therefore observers did not see any incorrect choices.

\section{Demonstrations for observers}

The observer group consisted of seven birds in order to be comparable with the sample size

424 in Miller and colleagues (2016): four females and three males. These individuals also 425 participated in Experiment 1: three from the trained group, three from the observer group, and 426 one from the control group. In an adjacent compartment with visual access to the observers, the 427 demonstrator lifted the demonstrated cup (white) and obtained the reward in four sessions, with 
42810 trials per session. Both cups were baited and could potentially be lifted, though the

429 demonstrator only lifted the demonstrated cup. The demonstrated cup location (left or right) was

430 counterbalanced across trials. Each observer watched one session per day.

431

432 Testing observers

433 After observers had seen Homer lifting the demonstrator cup 40 times, they were tested in 434 visual isolation from the group. Each observer was presented with the cups, both cups were 435 baited out-of-sight of the observer and we recorded which cup they touched first. They were 436 given one test trial, which lasted up to three minutes (all subjects interacted with the cups within

437 three minutes). They were allowed to touch both cups. The location of the demonstrated cup was 438 randomized across subjects. If they touched the demonstrated cup (white) first, we considered 439 this to be using social information from the demonstrator.

\section{Data analysis}

We recorded the colour and latency of the cup first touched by the demonstrator during training and demonstration trials, and by the observers during the test. The data were analysed using SPSS version 21 for the exact two-tailed Binomial tests, and R for the t-test. RM and KL both coded $20 \%$ of all videos across both experiments, with KL acting as a naïve coder, and inter-observer reliability was excellent (Cohen's kappa k=0.989, $\mathrm{p}<0.001$ ).

\section{Results}

Jays did not choose the demonstrated colour above chance levels (Binomial test: $p=0.453$ ).

450 Two of seven jays (one male, one female) chose the same coloured cup (white) as the 
451 demonstrator (i.e., copied the demonstrator), while the other five jays (three females, two males)

452 chose the non-demonstrated coloured cup (black; Table 3). In comparison, Miller, Schwab \&

453 Bugnyar (2016) found that eight of eight crows (five females, three males) and eight of eight

454 ravens (three females, five males) copied the conspecific demonstrator, which was significant

455 (Binomial test: $\mathrm{p}=0.008$ for each species). We additionally examined whether there was a

456 difference in the latency to make the first choice between the birds that chose the demonstrated

457 colour versus those that did not. The jays that chose the demonstrated colour did not have shorter

458 latencies to their first choice (Welch two-sample $\mathrm{t}$-test: $\mathrm{t}=0.88, \mathrm{p}=0.47, \mathrm{n}=7,95 \%$ confidence

459 interval=-36-57; data in ESM1 Table S1). We also explored whether relatedness influenced

460 likelihood to copy the demonstrator. Zero of two jays that selected the demonstrated coloured

461 cup (Binomial test: $\mathrm{p}=0.5, \mathrm{n}=2$ ) and two of five jays that did not select the demonstrated

462 coloured cup were siblings of the demonstrator bird (Binomial test: $\mathrm{p}=1.00, \mathrm{n}=5$ ). The birds did

463 not appear to show a group side bias because they did not select the cup on the same side

464 regardless of colour (Table 3: Binomial test: $p=1.00, n=7$ ).

465

466

\section{Discussion}

We found that relatively asocial Eurasian jays did not use social information (i.e.,

468 information made available by a conspecific) in the form of copying the choices of others in

469 either task. In Experiment 1 (object-dropping task), birds in the observer group first touched the

470 apparatus and object significantly sooner than birds in the control group, indicating a form of

471 social learning called stimulus enhancement. Stimulus enhancement attracts the attention of an

472 observer towards a specific object where the model acts (Giraldeau, 1997). However, observing a 
473 conspecific demonstrator did not facilitate solving the object-dropping task in Experiment 1, or

474 result in colour choice copying in Experiment 2.

475 Although corvids, including Eurasian jays, can be trained in the object-dropping task, it is

476 possible that this task is too difficult for social learning to facilitate the solution, except for the

477 occasional individual (i.e., one rook: Bird and Emery, 2009b; and one New Caledonian crow:

478 Mioduszewska, Auersperg \& von Bayern, 2015), thus masking whether Eurasian jays are able to

479 use social information by observing, and thus learning from, a demonstrator. In the present

480 experiment, solving this task required the observer birds to copy several actions of the

481 demonstrator: lifting the object from the table to insert into the tube to drop the collapsible

482 platform and obtain the reward, rather than just knocking an object into the tube from the tube

483 ledge, which is typically stage one of training. In further support of the suggestion that this task

484 is difficult for birds to learn is that only one bird has spontaneously solved the object-dropping

485 task in a previous study (one New Caledonian crow; Mioduszewska, Auersperg \& von Bayern,

486 2015), without any demonstrations or training. Additionally, birds, including corvids, typically

487 require a relatively large number of training trials to learn to solve this task, indicating that it is

488 fairly difficult to learn even with explicit training (e.g., 90-275 trials in the present experiment;

489 135-362 trials in great-tailed grackles: Logan, 2016; 76-255 trials in California scrub-jays: Logan

490 et al., 2016 - though note that definitions for reaching proficiency differ between these studies

491 and the current experiment).

492 It is therefore possible that the jays obtained some information from the demonstrator, but

493 potentially this information was not sufficient to enable them to complete the task (i.e., to insert

494 the object from the table into the tube). Therefore, we assessed whether there was any evidence

495 that the jays attended to the demonstrator, despite not being able to solve the task following the 
496 demonstrations, by measuring differences in the number of interactions with the apparatus and

497 object between the control and observer groups. Individuals in the observer group were not more

498 likely to touch the apparatus or object than individuals in the control group. Observer individuals

499 touched the apparatus and object in later trials more than control individuals, indicating that jays

500 may have been more persistent after having seen another bird solve the task. However, it should

501 be noted that the models showed only weak evidence for these two findings.

502 We also found that the observer group solved the object-dropping task significantly more

503 quickly than the trained group; however, there was no difference in the rate of learning (i.e., total

504 number of insertions required to solve the task) between the observer and control groups. The

505 strongest evidence of any form of social learning was in the form of stimulus enhancement:

506 observer birds that had seen a demonstrator interact with the apparatus and object first touched

507 these elements significantly sooner than control birds that had never observed another touching

508 the apparatus. It is possible that increased exposure to the apparatus may have facilitated learning

509 in both the observer and control groups, perhaps by removing neophobia of the apparatus

510 (although all birds were habituated to the apparatus prior to testing), and/or some social

511 facilitation of attraction or attention to the apparatus, as opposed to learning the actions to

512 perform the task. However, it is unclear whether observers attended to social information

513 provided by the conspecific or whether they would have learned about the task by observing a

514 'ghost control' where the object was inserted into the tube in the absence of a conspecific. Future

515 research incorporating ghost controls could distinguish between whether jays attend to social

516 information about what to attend to or whether they solely attend to the relevant object

517 movements and reward outcomes. 
In Experiment 2, in comparison with the object-dropping task, the colour discrimination task

519

520

521

522

523

524

525

526

527

528

529

530

531

532 was relatively simple as corvids are capable of making colour discriminations (Clayton \& Krebs, 1994; Range, Bugnyar \& Kotrschal, 2008). For example, there is evidence that juvenile Eurasian jays can discriminate between colours in similar two-choice discrimination tasks. Davidson and colleagues (Under Review) trained half of a group of Eurasian jays to associate a yellow coloured object with a reward and a green coloured object with no reward, and the other half to associate the green object with a reward and the yellow object with no reward. The jays then demonstrated proficiency by flying to the perch where the rewarded colour was located.

Further, the same task used in Experiment 2 was used previously in eight ravens and eight carrion crows, and all birds chose the demonstrated colour (Miller, Schwab \& Bugnyar, 2016). While the methods have some limitations (e.g., no counterbalancing of rewarded cup color, using only one demonstrator whose characteristics might have made him less likely for observers to attend to, low statistical power from only one trial per bird), we ran this task in a comparable manner to Miller, Schwab \& Bugnyar (2016) to allow for direct comparison between these two experiments, including the use of one male, same-age conspecific demonstrator to an observer group and one test trial. Additionally, all birds were hand-reared in species groups in a similar manner, tested by the same experimenter (RM) and similar sample sizes were used (eight ravens, eight crows, seven jays). We also similarly controlled for the influence of spatial location by randomizing the location of the demonstrated cup across subjects, and we found no group-level bias for one location (right/left) over the other (Table 3).

There were two notable differences between these experiments. Firstly, the colour discrimination task used different colours: blue and yellow cups in Miller, Schwab \& Bugnyar (2016) compared with white and black cups in the present experiment. The justification for this 
541 difference was the need to avoid a possible overlap between this experiment and the prior

542 experience of the jays with several different colours in differing reward scenarios during

543 previous studies (e.g., Davidson et al., Under Review). Furthermore, Shaw and colleagues (2015)

544 suggest that colour discrimination tasks should aim to use gray scale cues (e.g., light vs. dark

545 gray) to avoid innate species-level colour preferences. We cannot entirely rule out innate colour

546 preferences, because we did not transfer birds to novel colour combinations. However, innate

547 preferences would likely have been expressed at the species level, which did not occur here

548 because jays randomly chose white and black cups in their first trials.

549 Secondly, the jays were juveniles, whereas the ravens and crows were sub-adults. Therefore,

550 it is possible that social learning in the jays may not have developed by this early stage.

551 However, this is unlikely given that juveniles in other relatively asocial species exhibited social

552 learning whereas adults did not (Lupfer, Frieman \& Coonfield 2003; Noble, Byrne \& Whiting

553 2014). To our knowledge, no corvid studies have compared juvenile and adult social information

554 use. However, object permanence in Eurasian jays, which relates to caching development,

555 develops at a similar stage as in other corvids (ravens: Bugnyar, Stowe \& Heinrich, 2007;

556 California scrub-jays: Salwiczek et al., 2009). Specifically, jays reach a full (i.e., stage six

557 Piagetian) understanding of object permanence within their first few months of life (Zucca,

558 Milos \& Vallortigara, 2007). As the jays we tested were more than a few months of age, we do

559 not expect their behaviour to differ from adult behaviour with regard to social learning. The

560 finding that the jays behaved differently from the more social carrion crows and ravens in the use

561 of social information in this task is important. It raises the question of whether these more social

562 species - as with the more social rook (Bird \& Emery, 2009b) and New Caledonian crow 
563 (Mioduszewska, Auersperg \& von Bayern, 2015) - might be able to learn to copy the

564 demonstrator in the object-dropping task (Experiment 1).

565 Previous experiments have indicated that Eurasian jays do attend to social context in caching

566 and mate provisioning (Shaw \& Clayton, 2012; Shaw \& Clayton, 2013; Ostojić et al., 2013;

567 Shaw \& Clayton, 2014; Ostojić et al., 2014; Legg, Ostojić \& Clayton, 2016). It is therefore still

568 possible that jays use social information, but not for copying others' choices, as none of the

569 previous experiments required the birds to copy a demonstrator. Jays may also be more likely to

570 pay attention to and copy different demonstrators, such as an older, more affiliated or related

571 individuals, as model identity has been found to influence social learning in other corvids

572 (ravens, jackdaws: Schwab, Bugnyar \& Kotrschal 2008a; Schwab et al., 2008b). For example,

573 presence of siblings enhances social learning in ravens (Schwab et al., 2008b). Our demonstrator

574 was a sibling of some of the observers, which suggests that there was no influence of relatedness

575 to demonstrator on likelihood of copying in Experiment 2. However, our experiment was not

576 designed to test the relationship between relatedness and social learning and we do not have the

577 statistical power to make a firm conclusion on this point.

578 The use of social information is a process with several stages, which are likely to be

579 sequential and distinct: acquisition (observing another), application (performing the observed

580 behaviour, not necessarily successfully) and exploitation (successfully performing the observed

581 behaviour in a way that gives the individual an advantage; Carter, Tico \& Cowlishaw, 2016;

582 Guillette, Scott \& Healy, 2016). For instance, in chacma baboons (Papio ursinus), the average

583 individual acquired social information on $<25 \%$ of occasions and exploited social information on

$584<5 \%$ of occasions, and information use was dependent on phenotypic constraints such as network

585 position and dominance status (Carter, Tico \& Cowlishaw, 2016). The results of Experiments 1 
586 and 2 demonstrated that Eurasian jays did not appear to apply or exploit the social information

587 available even though they had the opportunity to acquire it. Although we reiterate that social

588 species also do not show a strong capacity to socially learn the object-dropping task in

589 Experiment 1.

590 In conclusion, Eurasian jays did not appear to use social information in the form of copying

591 the decisions of a conspecific in the object-dropping and colour discrimination tasks, which vary

592 in difficulty. However, their attention was drawn to the apparatus and object in the object-

593 dropping task as indicated by observers touching these components sooner than control birds. In

594 previous studies with social corvids, the birds had been explicitly tested for influences of social

595 information on learning the object-dropping task in only one study, with only one New

596 Caledonian crow learning the task following a conspecific demonstration (Mioduszewska,

597 Auersperg \& von Bayern, 2015). We also know that, when tested using very similar procedures,

598 including the same lead experimenter, ravens and crows use social information in the colour

599 discrimination task, in contrast to the jays. These corvid species vary in sociality, but all are

600 more social than the jays. Our results from relatively asocial Eurasian jays are therefore

601 consistent with those from relatively asocial Clark's nutcrackers (Bednekoff \& Balda 1996,

602 Templeton, Kamil \& Balda, 1999) in that social and relatively asocial corvids appear to differ in

603 their use of social information with regard to copying the choices of others. The present

604 experiment may indicate that Eurasian jays secondarily lost the ability to copy social information

605 provided by a conspecific, at least in some contexts, while maintaining the ability to attend to the

606 general movements of others, due to lack of selection pressure from an asocial environment.

607 However, more comparisons between social and relatively asocial corvids are needed to confirm

608 this hypothesis. 
609

610 Data Availability

611 Data for the social learning GLMMs is available at the KNB Data Repository at:

612 https://knb.ecoinformatics.org/\#view/corina_logan.45.5 (Miller \& Logan, 2016).

613

614 Acknowledgements

615 We thank Elsa Loissel and Natalie Williams for their help in the early stages of preparing for

616 Experiment 1 and discussion. Thank you to Maggie Dinsdale, Sam Melvin, Sarah Manley, Ivan

617 Vakrilov for animal care, to Ian Millar for help in apparatus construction, and to Mark Ghobain

618 for assistance in hand-rearing the Eurasian jays.

619

620

References

621 Balda, RP, Kamil AC, Bednekoff PA. 1996. Predicting cognitive capacity from natural history.

622 In Current ornithology (pp. 33-66). Springer US.

623 Bates D, Maechler M, Bolker B. 2011. lme4: Linear mixed-effects models using S4 classes. R 624 package version 0.999375-42. http://CRAN.R-project.org/package=lme4. Accessed 10 $625 \quad$ September 2016

626 Bednekoff PA, Balda RP. 1996. Observational spatial memory in Clark's nutcrackers and 627 Mexican jays. Animal Behaviour 52:,833-839

628 Bird CD, Emery NJ. 2009a. Rooks use stones to raise the water level to reach a floating worm. 629 Current Biology 19: 1410-4

630

Bird CD, Emery NJ. 2009b. Insightful problem solving and creative tool modification by captive 631 nontool-using rooks. Proceedings of the National Academy for Science 106: 10370-5 
632 Bird CD, Emery,NJ. 2010. Rooks perceive support relations similar to six-month-old babies. 633 Proceedings of the Royal Society of London B: Biological Sciences 277: 147-151

634 Bugnyar T, Stowe M, Heinrich B. 2007. The ontogeny of caching in ravens. Animal Behaviour $635 \quad 74: 757-767$

636 Burnham KP, Anderson DR. 2002. Model selection and multimodel inference: a practical 637 information- theoretic approach, 2nd edn. New York, NY: Springer.

638 Carter AJ, Tico MT, Cowlishaw G. 2016. Sequential phenotypic constraints on social 639 information use in wild baboons. eLife 5: e13125

640 Cheke LG, Bird CD, Clayton NS. 2011. Tool-use and instrumental learning in the Eurasian jay. Animal Cognition 14: 441-455

642 Clayton NS, Dally JM, Emery NJ. 2007. Social cognition by food-caching corvids: the western 643 scrub-jay as a natural psychologist. Philosophical Transactions of the Royal Society of London 362: 507-522

Clayton NS, Dickinson A. 1998. Episodic-like memory during cache recovery by scrub jays. Nature 395: 272-278.

647 Clayton NS, Emery NJ, Dickinson A. 2006. The prospective cognition of food caching and recovery by Western scrub-jays. Comparative Cognition \& Behavior 1: 1-11

Clayton NS, Emery NJ. 2007. The social life of corvids. Current Biology 17: R652-656

Clayton NS, Krebs JR. 1994. Memory for spatial and object-specific cues in food-storing and non-storing species of birds. Journal of Comparative Physiology A 174, 371-379. 
654 Davidson G, Miller R, Loissel E, Cheke L, Clayton N (Under Review). Development of support $655 \quad$ intuitions in juvenile Eurasian jays

656 de Kort SR, Clayton NS. 2006. An evolutionary perspective on caching by corvids. Proceedings 657 of the Royal Society of London Series B 273: 417-423

658 Emery NJ, Clayton NS. 2004. The mentality of crows: Convergent evolution of intelligence in $659 \quad$ corvids and apes. Science 306:1903-1907

660 Emery NJ, Clayton NS. 2005. Evolution of avian brain and intelligence. Current Biology 15: $661 \quad$ R946-950

662 Fritz J, Kotrschal K. 1999. Social learning in common ravens. Animal Behaviour 57:785-793

663 Garrone Neto D, Uieda VS. 2012. Activity and habitat use of two species of stingrays

664 (Myliobatiformes: Potamotrygonidae) in the upper Paraná River basin, Southeastern 665 Brazil. Neotropical Ichthyology 10: 81-88

666 Giraldeau LA. 1997. The ecology of information use. In: Behavioural Ecology (Krebs JR, Davis 667 NB, eds). Oxford: Blackwell Science

668 Goodwin D. 1951. Some aspects of the behavior of the jay. Ibis 93: 414-442

669 Goodwin D. 1986. Crows of the world. Ithaca, NY: Cornell University Press

670 Guillette LM, Scott ACY, Healy SD. 2016. Social learning in nest-building birds: a role for 671 familiarity. Proceedings of the Royal Society: Biological Sciences 283: 20152685

672 Hunt GR. 1996. Manufacture and use of hook-tools by New Caledonian crows. Nature 379: 249$673 \quad 51$

674 Jelbert SA, Taylor AH, Cheke LG, Clayton NS, Gray RD. 2014. Using the Aesop's Fable 675 paradigm to investigate causal understanding of water displacement by New Caledonian

676 crows. PLoS ONE 9: e92895 
677 Jelbert SA, Taylor AH, Gray RD. 2015. Reasoning by exclusion in New Caledonian crows

678 (Corvus moneduloides) cannot be explained by avoidance of empty containers. Journal of

679 Comparative Psychology 129: 283

680 Kuznetsova A, Brockhoff PB, Christensen RHB. 2015. lmerTest: Tests in linear mixed effects

681 models. R package. https://cran.rproject.org/web/packages/lmerTest/index.html Accessed

$682 \quad 10$ September 2016.

683 Lefebvre L, Giraldeau LA. 1996. Is social learning an adaptive specialization? In: Social

684 Learning in Animals: The Roots of Culture. Heyes, Cecilia M., and Bennett G. Galef Jr, 685 eds. Academic Press, Inc.

686 Legg EW, Clayton NS. 2014. Eurasian jays conceal caches from onlookers. Animal Cognition

$687 \quad 17: 1223-1226$

688 Legg EW, Ostojić L, Clayton NS. 2016. Caching at a distance: a cache protection strategy in $689 \quad$ Eurasian jays. Animal Cognition 19: 753-758.

690 Logan CJ. 2015. Innovation does not indicate behavioral flexibility in great-tailed grackles. $691 \quad$ BioRxiv

692 Logan CJ. 2016. Behavioral flexibility and problem solving in an invasive bird. Peer J 4: e1975

693 Logan CJ, Breen A, Taylor, AH, Gray RD, Hoppitt WJE. 2016. How New Caledonian crows 694 solve novel foraging problems and what it means for cumulative culture. Learning and 695 Behavior 44: 18-28.

696 Logan CJ, Harvey BD, Schlinger BA, Rensel M. 2016. Western scrub-jays do not appear to 697 attend to functionalist in Aesop's Fable experiments. Peer J 4: e1707

698 Logan CJ, Jelbert SA, Breen A, Gray RD, Taylor AH. 2014. Modifications to the Aesop's Fable 699 paradigm change New Caledonian crow performances. PLoS ONE 9: e103049 
700

701

702

703

704

705

706

707

708

709

710

711

712

713

714

715

716

717

718

719

720

721

Lupfer G, Frieman J, Coonfield D. 2003. Social transmission of flavor preferences in two species of hamsters (Mesocricetus auratus and Phodopus campbelli). Journal of Comparative Psychology 117: 449

Miller R, Logan CJ. 2016. Data for: Eurasian jays do not use social information provided by a conspecific model. KNB Data Repository. https://knb.ecoinformatics.org/\#view/corina_logan.45.5

Miller R, Schwab C, Bugnyar T. 2016. Explorative innovators and flexible use of social information in common ravens and carrion crows. Journal of Comparative Psychology

Mioduszewska B, Auersperg B, von Bayern AMP. 2015. Jackdaws, crows, and stones - social learning of a stone tool-use task. International Ethological Conference, Cairns, Australia

Noble DW, Byrne RW, Whiting MJ. 2014. Age-dependent social learning in a lizard. Biology letters 10: 20140430

Ostojić L, Legg EW, Shaw RC, Cheke LG, Mendl M, Clayton NS. 2014. Can male Eurasian jays disengage from their own current desire to feed the female what she wants? Biology letters 10: 20140042

Ostojić L, Shaw RC, Cheke L, Clayton NS. 2013. Evidence suggesting that desire-state attribution may govern food sharing in Eurasian jays. Proceedings of the National Academy for Science 110: 4123-4128

Raby CR, Alexis DM, Dickinson A, Clayton NS. 2007. Planning for the future by Western Scrub-Jays. Nature 445: 919-921.

Range F, Bugnyar T, Kotrschal K. 2008. The performance of ravens on simple discriminations tasks: a preliminary study. Acta Ethologica $11: 34-41$ 
722 R Core Team. 2015. R: a language and environment for statistical computing. Vienna: R

723

724

725

726

727

728

729

730

731

732

733

734

735

736

737

738

739

740

741

742

743

Foundation for Statistical Computing. Available at https://www.R-project.org (accessed on 10 September 2016).

Salwiczek LH, Emery NJ, Schlinger BA, Clayton NS. 2009. The development of caching and object permanence in Western scrub-jays: which emerges first? Journal of Comparative Psychology 123: 295-303

Schwab C, Bugnyar T, Kotrschal K. 2008a. Preferential learning from non-affiliated individuals in jackdaws. Behavioural Processes 79:148-55

Schwab C, Bugnyar T, Schloegl C, Kotrschal K. 2008b. Enhanced social learning between siblings in common ravens. Animal Behaviour 75: 501-508

Seed AM, Clayton NS, Emery NJ. 2008. Cooperative problem solving in rooks (Corvus frugilegus). Proceedings of the Royal Society of London B: Biological Sciences 275: $1421-1429$

Shaw RC, Boogert NJ, Clayton NS, Burns KC. 2015. Wild psychometrics: Evidence for 'general' cognitive performance in New Zealand robins. Animal Behaviour 109: 101-111

Shaw RC, Clayton NS. 2012. Eurasian jays flexibly switch caching and pilfering tactics in response to social context. Animal Behaviour 84:1191-1200

Shaw RC, Clayton NS. 2013. Careful cachers and prying pilferers: Eurasian jays (Garrulus glandarius) limit auditory information available to competitors. Proceedings of the Royal Society of London B: Biological Sciences 280: 20122238

Shaw RC, Clayton NS. 2014. Pilfering Eurasian jays use visual and acoustic information to locate caches. Animal Cognition 17: 1281-8. 
744 Snow DW, Perrins C. 1997. The birds of the Western Palearctic. Oxford University Press:

$745 \quad$ Oxford

746 St Clair JJH, Burna ZT, Bettaney EM, Morrissey MB, Burt J, Otis B, Ryder TB, Fleischer RC,

747 James R, Rutz C (2015). Experimental resource pulses influence social-network

748 dynamics and the potential for information flow in tool-using crows. Nature

$749 \quad$ Communications 6: 7197

750 Taylor AH, Miller R, Gray RD. 2012. New Caledonian crows reason about hidden causal agents.

751 Proceedings of the National Academy of Sciences 109: 16389-16391

752 Templeton JJ, Kamil AC, Balda RP. 1999. Sociality and social learning in two species of

753

754 (Nucifraga columbiana). Journal of Comparative Psychology 113: 450

755 Thonhauser KE, Gutnick T, Byrne RA, Kral K, Burghardt GM, Kuba MJ. 2013. Social learning

756 in Cartilaginous fish (stingrays Potamotrygon falkneri). Animal cognition 16: 927-932

757 Valone TJ, Templeton JJ. 2002. Public information for the assessment of quality: a widespread

758 social phenomenon. Philosophical Transactions of the Royal Society of London B:

759 Biological Sciences 357: 1549-1557

760 Wilkinson A, Kuenstner K, Mueller J, Huber L. 2010. Social learning in a non-social reptile 761 (Geochelone carbonaria). Biology Letters: rsbl20100092

762 Zucca P, Milos N, Vallortigara G. 2007. Piagetian object permanence and its development in $763 \quad$ Eurasian jays. Animal Cognition 10: 243-258 


\section{Table $\mathbf{1}$ (on next page)}

Stages of the object insertion apparatus.

Training stages: training the trained group, and subsequent to their tests, the observer and control groups, to insert objects into the tube to release the food reward. Training stages occurred in the following sequence: 1-2-3. Demonstrator stages: birds in the observer group watched the demonstrator solve the apparatus 40 times per stage before being presented with the final stage apparatus in a test trial. Demonstration stages occurred in the following sequence: 3-1-2-3. 
1 Table 1. Stages of the object insertion apparatus. Training stages: training the trained group, and

2 subsequent to their tests, the observer and control groups, to insert objects into the tube to release

3 the food reward. Training stages occurred in the following sequence: 1-2-3. Demonstrator stages:

4 birds in the observer group watched the demonstrator solve the apparatus 40 times per stage

5 before being presented with the final stage apparatus in a test trial. Demonstration stages

6 occurred in the following sequence: 3-1-2-3.

\begin{tabular}{|c|c|c|c|c|c|}
\hline \multirow[t]{2}{*}{ Stage } & \multicolumn{2}{|c|}{$\begin{array}{c}\text { Removable platform } \\
\text { position }\end{array}$} & \multicolumn{2}{|c|}{ Object position } & \multirow{2}{*}{$\begin{array}{l}\text { Figure } 2 \\
\text { corresponding } \\
\text { image }\end{array}$} \\
\hline & Training & Demonstration & Training & Demonstration & \\
\hline 1 & $\begin{array}{l}\text { Top of the } \\
\text { tube }\end{array}$ & Top of the tube & $\begin{array}{l}\text { Platform. } \\
\text { Object } \\
\text { baited with } \\
\text { insect and } \\
\text { then not } \\
\text { baited }\end{array}$ & Table & $\mathrm{a}$ \\
\hline 2 & $\begin{array}{l}\text { Gradually } \\
\text { lowered } \\
\text { down the } \\
\text { tube using } \\
\text { plastic } \\
\text { rings until } \\
\text { at the } \\
\text { bottom of } \\
\text { the tube }\end{array}$ & $\begin{array}{l}\text { At the bottom of } \\
\text { the tube }\end{array}$ & $\begin{array}{l}\text { Platform or } \\
\text { table }\end{array}$ & Table & $\mathrm{b}$ \\
\hline 3 & $\begin{array}{l}\text { No } \\
\text { platform }\end{array}$ & No platform & $\begin{array}{l}\text { Apparatus } \\
\text { base or table }\end{array}$ & Table & $\mathrm{c}$ \\
\hline
\end{tabular}

7

8 


\section{Table 2 (on next page)}

Did observers learn what to attend to from the demonstrator?

Results from the GLM (Model 1) and GLMM (Model 2) examining whether individuals in the observer group touched the apparatus and object more than control individuals (Model 1) or whether they interacted more with particular parts of the apparatus (base or tube) or object (Model 2). Model 3 (GLMM) examined latencies to first touch per trial to determine whether individuals in the observer group first touched the apparatus/object sooner than control birds. SE: standard error, $z: z$ value, $p: p$ value, the rows in italics list the variance and standard deviation of the random effect. 
1 Table 2. Results from the GLM (Model 1) and GLMM (Model 2) examining whether individuals

2 in the observer group touched the apparatus and object more than control individuals (Model 1)

3 or whether they interacted more with particular parts of the apparatus (base or tube) or object

4 (Model 2). Model 3 (GLMM) examined latencies to first touch per trial to determine whether

5 individuals in the observer group first touched the apparatus/object sooner than control birds. SE:

6 standard error, $\mathrm{z}$ : $\mathrm{z}$ value, $\mathrm{p}$ : $\mathrm{p}$ value, the rows in italics list the variance and standard deviation of

7 the random effect.

\begin{tabular}{llllll}
\hline Model & Variable & Estimate & SE & z & p \\
\hline 1 & Intercept (controls) & 3.19 & 0.17 & 18.42 & $<0.001$ \\
& Trial & -0.37 & 0.07 & -5.62 & $<0.001$ \\
& Observers & -0.17 & 0.21 & -0.83 & 0.41 \\
& Trial*Observers & 0.16 & 0.08 & 2.06 & 0.04 \\
\hline 2 & Intercept (apparatus & 1.19 & 0.25 & 4.83 & $<0.001$ \\
& base, controls) & & & & \\
& Object & -0.25 & 0.20 & -1.12 & 0.23 \\
& Tube & -0.32 & 0.21 & -1.54 & 0.12 \\
& Observers & 0.44 & 0.29 & 1.50 & 0.13 \\
& Observers*object & -0.37 & 0.24 & -1.51 & 0.13 \\
& Observers*tube & -0.14 & 0.24 & -0.59 & 0.56 \\
& Bird ID & 0.12 & 0.35 & & \\
\hline 3 & Intercept (controls) & 4.32 & 0.21 & 20.88 & $<0.001$ \\
& Observers & -1.22 & 0.26 & -4.78 & $<0.001$ \\
& Bird ID & 0.13 & 0.35 & & \\
\hline
\end{tabular}

8 


\section{Table 3(on next page)}

Two-choice colour discrimination task results

The birds observed the trained demonstrator Homer lifting the white cup to retrieve a mealworm on 40 consecutive trials. 
1 Table 3. Two-choice colour discrimination task results. The birds observed the trained

2 demonstrator Homer lifting the white cup to retrieve a mealworm on 40 consecutive trials.

\begin{tabular}{llllll}
\hline ID & Sex & $\begin{array}{l}\text { Demonstrated } \\
\text { colour }\end{array}$ & $\begin{array}{l}\text { Chosen } \\
\text { colour (first } \\
\text { choice) }\end{array}$ & $\begin{array}{l}\text { Location of } \\
\text { chosen colour }\end{array}$ & $\begin{array}{l}\text { Latency to } \\
\text { first choice } \\
\text { (s) }\end{array}$ \\
\hline Dolci & F & White & Black & Left & 19 \\
Stuka & F & White & Black & Right & 51 \\
Horatio & M & White & White & Left & 44 \\
Booster & M & White & Black & Left & 20 \\
Lintie & F & White & Black & Right & 12 \\
Gizmo & F & White & White & Right & 25 \\
Roland & M & White & Black & Left & 19 \\
\hline
\end{tabular}

3

4 


\section{Figure 1}

Experiment 1 set up: Stages of the object insertion apparatus.

a) The removable platform at the top of the tube, b) the removable platform at the bottom of the tube, and c) the final stage apparatus (no removable platform). Photo: Rachael Miller.

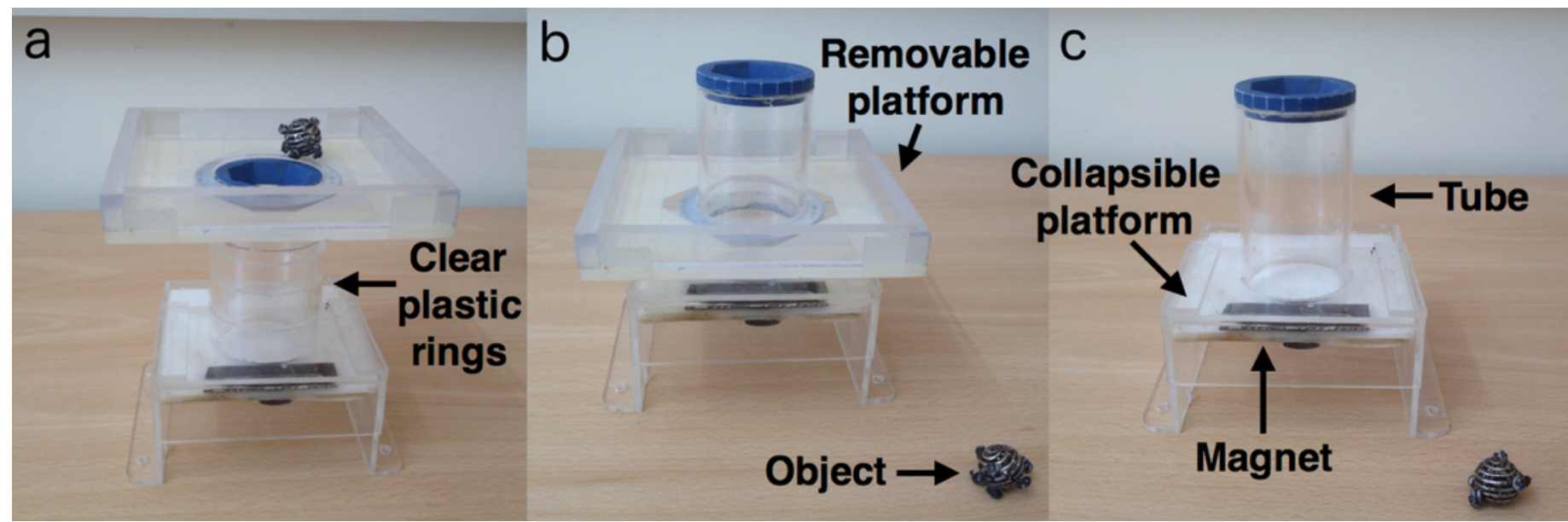




\section{Figure 2}

Experiment 2 set up.

Two-choice colour discrimination task where observers only saw a demonstrator find food under the white cup. Photo: Sarah Jelbert.

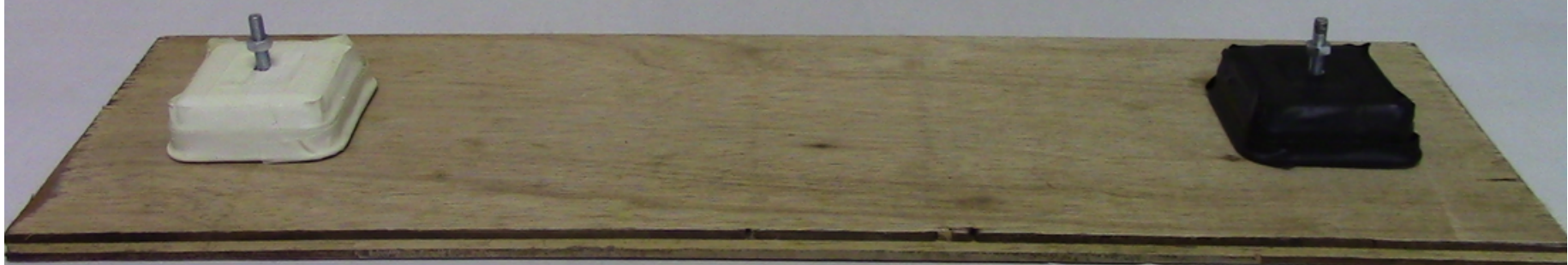


Figure 3

Experiment 1: Object dropping test trials for observer and control groups

Mean latency to first touch of the apparatus or object per trial for Observer (white boxplot) and Control (hatched boxplot) groups.

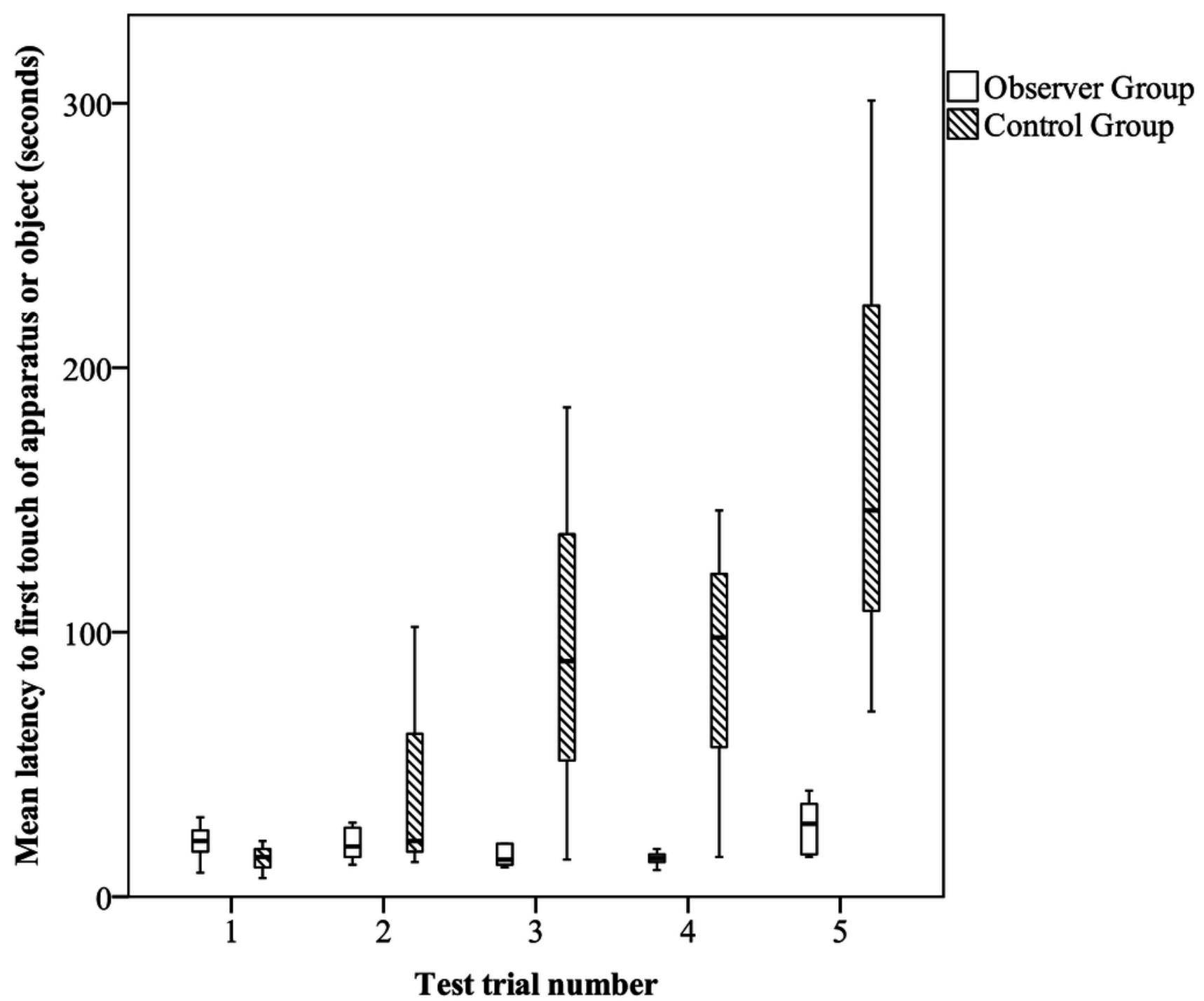


Figure 4

Experiment 1: number of object insertions to solve

Total number of object insertions to solve the object-dropping task per group

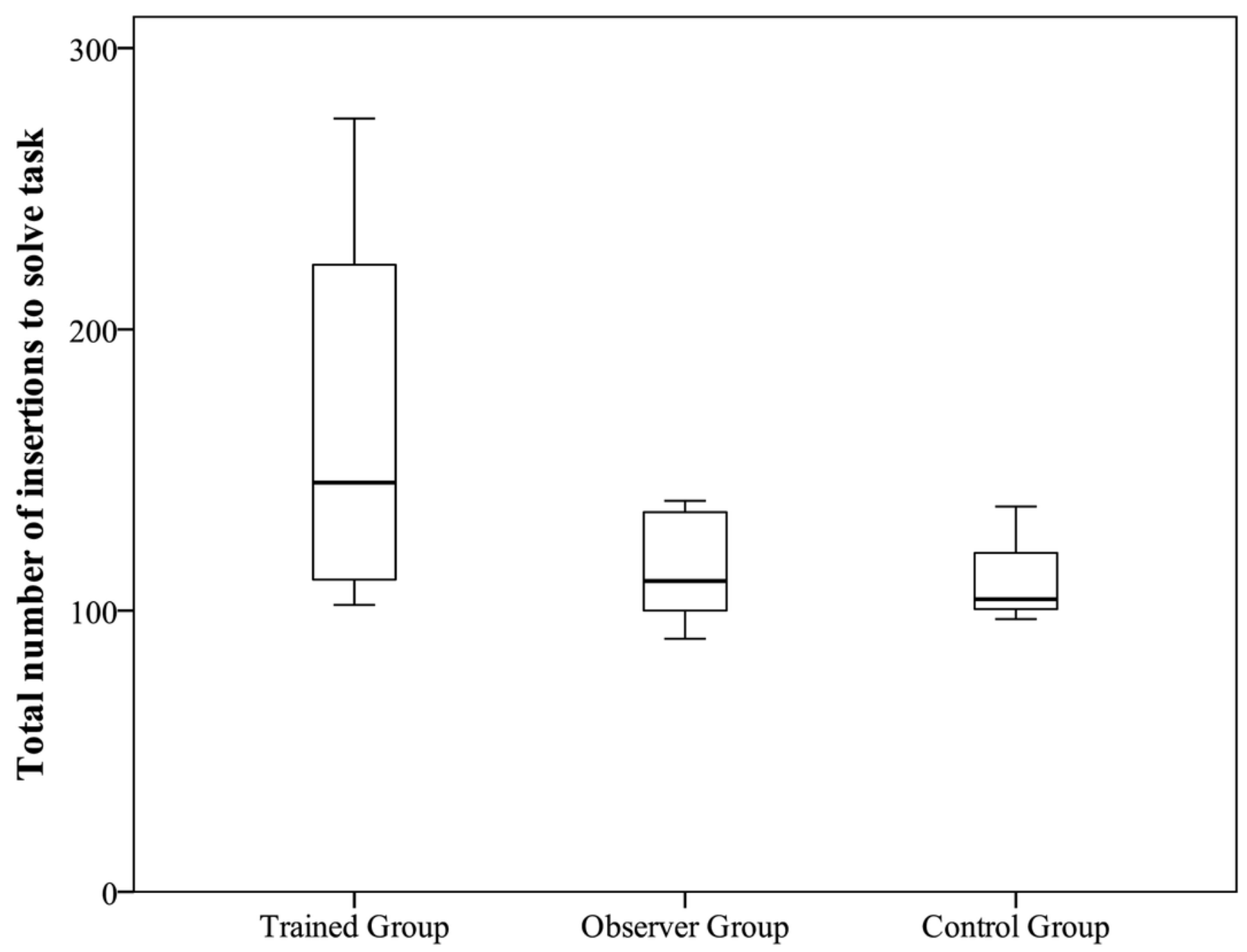

